\title{
Electrochemistry on tribocharged polymers is governed by the stabil- ity of surface charges rather than charging magnitude
}

Jinyang Zhang, ${ }^{\dagger}$ Fergus J. M. Rogers, ${ }^{\ddagger}$ Nadim Darwish, ${ }^{\dagger}$ Vinicius R. Gonçales, ${ }^{\circledR}$ Yan B. Vogel, ${ }^{\dagger}$ Fei Wang, ${ }^{\dagger}$ J. Justin Gooding, ${ }^{\S}$ M. Chandramalika. R. Peiris, ${ }^{\dagger}$ Guohua Jia, ${ }^{\dagger}$ Jean-Pierre Veder, ${ }^{\|}$Michelle L. Coote, ${ }^{*}, \neq$ and Simone Ciampi ${ }^{*}, \dagger$

${ }^{\dagger}$ School of Molecular and Life Sciences, Curtin Institute of Functional Molecules and Interfaces, Curtin University, Bentley, Western Australia 6102, Australia

${ }^{\ddagger}$ ARC Centre of Excellence for Electromaterials Science, Research School of Chemistry, Australian National University, Canberra, Australian Capital Territory 2601, Australia

§School of Chemistry, The Australian Centre for NanoMedicine and the Centre of Excellence in Convergent Bio-Nano Science and Technology, The University of New South Wales, Sydney, New South Wales 2052, Australia

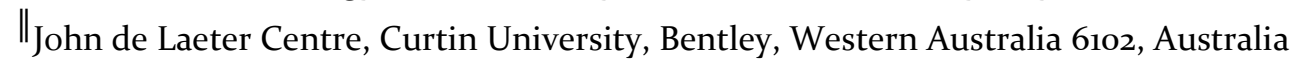

ABSTRACT: Electrically insulating objects gain a net electrical charge when brought in and out of contact. This phenomenon - triboelectricity - involves the flow of charged species, but to conclusively establish their nature has proven extremely difficult. Here, we demonstrate an almost linear relationship between a plastic sample's net negative charge and the amount of solution metal ions discharged to metallic particles, with a coefficient of proportionality linked to its electron affinity (stability of anionic fragments). The maximum magnitude of reductive redox work is also material-dependent: metallic particles grow to a larger extent over charged dielectrics that yield stable cationic fragments (smaller ionization energy). Importantly, the extent to which the sample can act as electron source greatly exceeds the net charging measured in a Faraday pail/electrometer set up, which brings direct evidence of triboeletricity being a mosaic of positive and negative charges rather than a homogenous ensemble, and defines for the first time their quantitative scope in electrochemistry.

\section{INTRODUCTION}

Materials that are poor conductors of electricity can nonetheless gain an excess of electrical charges. Contact electrification of dielectric materials that are first put into contact and then separated is a familiar topic; examples range from children's hair electrified by a party balloon, explosive discharges, ${ }^{1}$ to the $\$ 250$ billion market of office toners, copiers and laser printers. ${ }^{2}$ Despite our familiarity with the phenomenon, we are still without a complete chemical picture of its origin. ${ }^{3}$ Several mechanisms appear to be involved in the generation of charges upon contact: the exact chemical details of the surfaces involved are of importance, ${ }^{4}$ the participation of ions derived from water appears to be relevant, ${ }^{5}$ and there is a relationship between friction fluctuations, ${ }^{6}{ }^{\text {cracking }}{ }^{7}$ and tribocharging. As such the entire picture is extremely complex, all in all making it difficult to get a coherent picture on the charging part of the phenomenon. ${ }^{8}$
What is even more striking is the intense scientific debate around the nature of the charge carriers, with ongoing controversy between models explaining charging upon contact either transfer of ions ${ }^{9}$ or transfer of electrons.$^{10}$ Regardless, charging occurs, and large charge densities can be obtained, especially on rough surfaces, ${ }^{11}$ leading to the possibility of large electric fields adjacent to the tribocharged material. ${ }^{12}$ Moreover, while the emission of charged particles by strained or abraded dielectrics is a transient phenomenon and it decays rapidly after contact, ${ }^{13}$ a large fraction of tribocharges, electron or ions, are known to survive at defect sites for relatively long period of times. ${ }^{14}$ There is therefore a scope for tribocharged interfaces in delivering a precise amount of charge (Coulombs) to a liquid or gas sample, ${ }^{15}$ and the possibility of using electrical insulators to guide redox $^{16}$ and potentially non-redox chemical changes. ${ }^{17}$

The overall net charging of a polymer sample can be measured directly and with great accuracy in a Faraday pail. However, what is measured is the arithmetic sum of net negative/net positive local surface-domains, ${ }^{18}$ in other 
words, a statically-charged sample that bears a net charge of $-1 \mathrm{nC}$, the equivalent charge of $6.24 \times 10^{9}$ electrons, is possibly carrying a much larger number of species having a negative charge. Recent measurements of charging with sub-micrometre lateral resolution have in fact demonstrated that tribocharged plastics can accommodate significantly more charge per unit area than previously considered possible, ${ }^{\mathrm{b}}$ opening up possibilities in the use of charged dielectrics to trigger or catalyze chemical reactions on a practical, or at least measurable, scale.

Here we provide a quantitative understanding of the extent to which electrochemical work can be harnessed from nonelectroneutral dielectrics. We have quantified metal deposition on electrostatically-charged polydimethylsiloxane (PDMS), polytetrafluoroethylene (PTFE), polyvinyl chloride (PVC), and nylon samples that were charged to either net positive or net negative Coulomb values. X-ray photoelectron spectroscopy (XPS), atomic force (AFM) and transmission electron (TEM) microscopies, as well as electron diffraction and quantum chemical methods are used to gauge the practical electrochemical implications of contact electrification being a "mosaic" 9 b, 18 of polymer fragments of both negative and positive charge.

\section{RESULTS AND DISCUSSION}

At the nanoscale it is impossible to preclude the rupture of chemical bonds upon contact ${ }^{20}$ and electrons are known to be rapidly emitted from a number of materials in response to fracture, including insulators, glass, graphite and polymers. ${ }^{21}$ While this type of energy dissipation in a material that is under physical stress is generally regarded as topic for tribochemistry - a good example is the chemical changes experienced by lubricants in a ball mill ${ }^{22}-$ in this work we focused instead on residual charges that persist on a plastic sample several minutes after it is separated from another dielectric: care is taken to minimize forceinduced effects on bonds and bonding, ${ }^{23}$ and to clarify on the nature of charged species guiding redox reactivity on tribocharged insulators.

Our Faraday pail data show that for PDMS and PTFE samples only about $20 \%$ and $23 \%$ (respectively) of tribocharges have dissipated $2000 \mathrm{~s}$ after the separation from a glass surface (Supporting Information, Figure $\mathrm{S} 1$ ). The kinetics of the charge decay of PTFE samples was also measured by Kelvin probe force microscopy (KPFM, Supporting Information, Figure $\mathrm{S}_{2}$ ) and found consistent with the time-resolved Faraday pail data. A faster decay is observed for PVC samples, losing about $75 \%$ of their negative charges over the same period of time (Figure $\mathrm{S}$ ). Charge dissipation in air is faster for PVC than for PTFE, an observation that appears to be linked to the lower work function of the former (5.13 eV, ${ }^{24}$ PVC; $5.75 \mathrm{eV},{ }^{24}$ PTFE). Direct experimental measurements of work function in dielectrics suffer from a range of drawbacks, and data available are limited. However, a relationship exists between the work function of a polymer and the stability of its cationic fragments: it can be approximated as one-half of the ionization energy (IE)

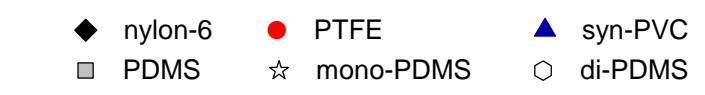

(a)

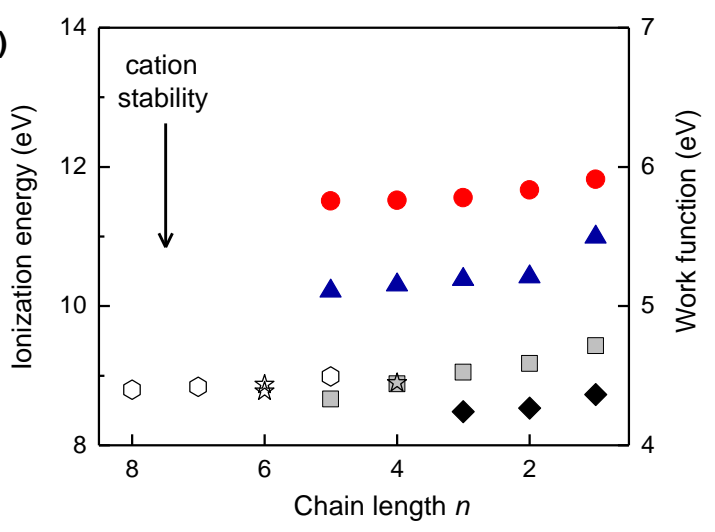

(b)

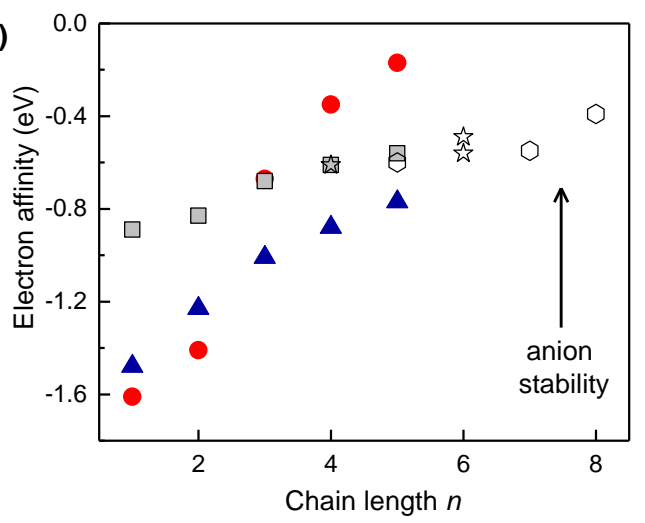

Figure 1. (a) Computed $298 \mathrm{~K}$ ionization energies (IE) and (b) electron affinities (EA) for oligomers of PTFE, synPVC, nylon-6 and PDMS as a function of the chain length $n$ (Supporting Information, Table S1). Structures and benchmarking calculations are provided in the Supporting Information (Figure S3 and Table S2, respectively). Note that the sign conventions for IE and EA are such that a larger IE implies a less stable cation, whereas a higher (less negative) EA implies a more stable anion. For linear polymers, the work function was approximated as one-half of the ionization energy at $n=1(4.38 \mathrm{eV}, 5.93 \mathrm{eV}$ and 5.51 $\mathrm{eV}$ for nylon, PTFE and PVC, respectively). For branched polymers this approximation does not hold and so a theoretical value for the work function cannot be computed (experimental data available for silicone oil is $\geq 6.71 \mathrm{eV}^{19}$ ).

of its unimeric oligomer ${ }^{24}$ (one-half of IE when $n=1$, right $y$-axis in Figure 1a). Computed IE gas phase values for oligomers of the linear dielectric materials used are in Figure 1 , and under the assumptions of (i) polymer fragments being the charge carrier in a tribocharged plastic, and (ii) equally stable anions, a lower IE - more stable cations would account for PVC losing in air its net negative charge more rapidly than PTFE. However, the stability of anions is also expected to be of relevance in the discharge process, and this becomes apparent when the polymers are discharged in solution of silver ions (vide infra). Hence the stability of anions are in the order PTFE $>$ PDMS $>$ PVC (at the longest chain length studied, $n=5$ ), so in PVC both the stability of its cations and the instability of its anions - 

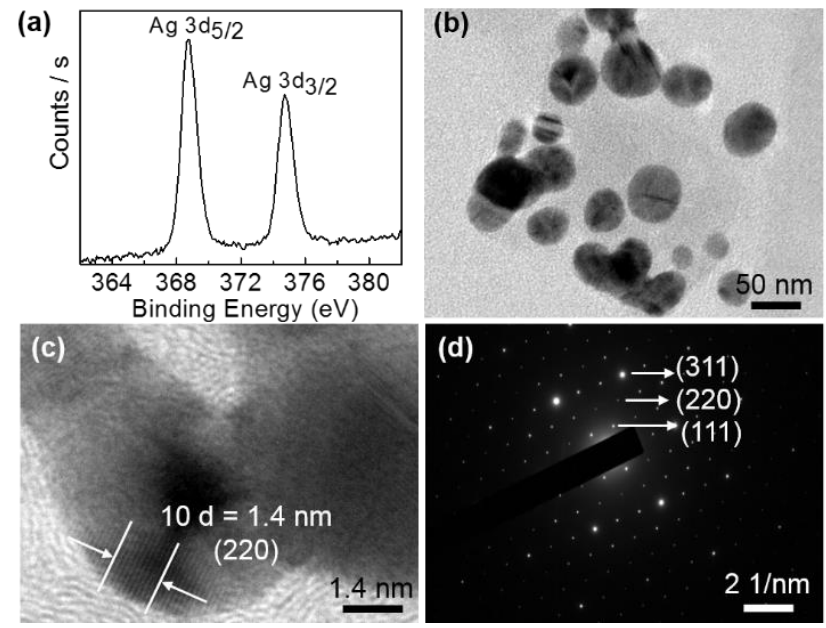

Figure 2. Analysis of silver nanoparticles electrochemically grown on electrical insulators (tribocharged PDMS samples immersed in aqueous $\mathrm{AgNO}_{3}$ solutions). (a) XPS analysis of the PDMS sample indicates an Ag $3 d$ spinorbit-split emissions consistent with literature values for metallic silver $\left(\mathrm{Ag} 3 \mathrm{~d}_{3 / 2}\right.$ and $\mathrm{Ag} 3 \mathrm{~d}_{5 / 2}$ at $374.4 \mathrm{eV}$ and 368.4 $\mathrm{eV}$, respectively). (b) TEM image of $\mathrm{Ag}$ particles showing sizes around $50 \mathrm{~nm}$ in diameter. (c) HRTEM image indicating a lattice spacing of $0.14 \mathrm{~nm}(10 \mathrm{~d}=1.4 \mathrm{~nm})$, corresponding to fcc Ag, and matching the spacing of (220) lattice planes. (d) Selected area electron diffraction (SAED) patterns, with labels indicating the (hkl) assignments.

low positive IE and large negative EA - are in line with the faster discharge observed: tribocharges are the result of the presence of charged polymer fragments.

Guided by our data on discharge in air, and by the 2008 proof-of-concept by Liu and Bard, describing the growth of metal films on tribocharged PTFE, ${ }^{16 \mathrm{~b}}$ we ran XPS, TEM and selected area electron diffraction (SAED) tests on charged plastic samples that were discharged by immersion in chloroauric acid solutions. TEM and SAED data recorded ex situ from a single particle (Supporting Information, Figure S4a,b) exhibits a diffraction pattern with indexed planes of (422), (220) and (111), which is in very good agreement with literature diffraction patterns for gold crystals. ${ }^{25}$ Under particular conditions, such as under the strong electric field of a water-air interface, ${ }^{26}$ the reduction of gold ions can occur spontaneously, without a reducing agent or an applied bias. ${ }^{27}$ Therefore, our observation of metallic gold nanoparticles growing on an insulator such as PDMS after a tribo charge/discharge sequence, as supported by XPS, TEM and SAED data in Supporting Information, Figure $S_{4}$, is unexpected but yet not a direct and conclusive evidence of a redox role played by tribocharges - electrons or ions. Silver ions are thermodynamically more stable than gold ones. The redox potential for the reduction of isolated ions or for the addition of metallic silver on small clusters is very negative, ${ }^{28}$ possibly as low as $-1.8 \mathrm{~V}$ vs SHE for isolated ions, and close to o V vs SHE for micrometre-sized clus- (a)
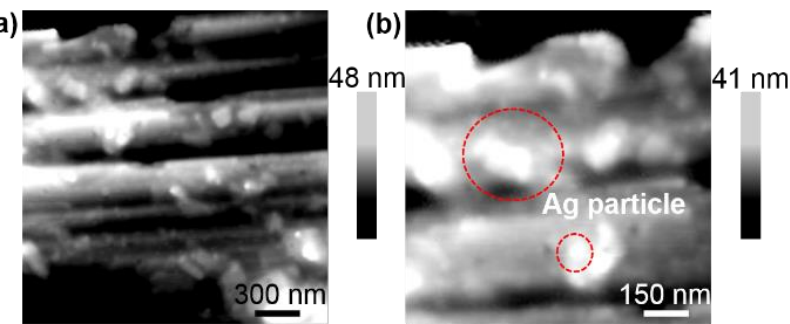

(c)
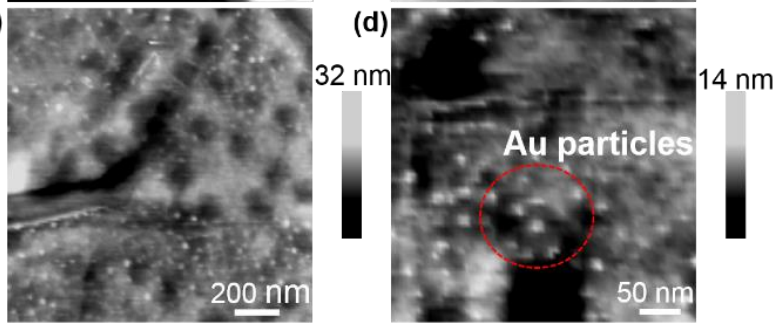

Figure 3. AFM topography images of silver (a), (b) and gold (c), (d) nanoparticles grown on tribocharged PDMS samples. AFM data indicates and approximate diameter for the silver particles, or cluster of particles (red circles), between 50 and $150 \mathrm{~nm}$, and dimeters for gold particles between 10 and $20 \mathrm{~nm}$.

ters. ${ }^{29}$ Analogously to the experiments done with chloroauric acid, we immersed tribocharged samples of PDMS in solutions of silver nitrate. The spectroscopic analysis of nine independently prepared and analyzed samples showed consistently two Ag $3 \mathrm{~d}$ spin-orbit-split XPS emissions (Figure 2a; $\mathrm{Ag} 3 \mathrm{~d}_{3 / 2}$ and $\mathrm{Ag} 3 \mathrm{~d}_{5 / 2}$ at $374.4 \mathrm{eV}$ and 368.4 $\mathrm{eV}$, respectively) of spectral position consistent with literature values for metallic silver..$^{30}$ Adventitious spectral shifts were accounted for, and the positions of $C-C$ and $C-$ F bands match their literature value of $284.8 \mathrm{eV}$ and 292.2 $\mathrm{eV}$, respectively (Supporting Information, Figure $\mathrm{S}_{5}$ ). ${ }^{31}$

Examination by TEM of these silver nanostructures (Figure 2b) revealed a diameter of ca. $50 \mathrm{~nm}$ and a lattice structure with an interplanar spacing of $1.44 \AA$ (HRTEM, Figure 2c), which matches with the spacing of the (220) lattice planes of Ag. ${ }^{32}$ These nanostructures were subjected to selected area electron diffraction (SAED, Figure 2d) and diffraction patterns recorded from individual particles suggest a polycrystalline nature, with evidence of the (111), (220) and (311) reflections of fcc silver. 33

The initial amount of net charging was recorded for each sample prior to the discharge step and was generally found to be between -1 and $-8 \mathrm{nC} \mathrm{cm}{ }^{-2}$, which is the equivalent of only $0.6-5 \times 10^{10} \mathrm{~cm}^{-2}$ elementary charges. The amount of silver present on the sample after the discharge, as measured by XPS, was surprisingly large, possibly as high as $10^{15}$ atoms $\mathrm{cm}^{-2}$ (Supporting Section 1 and Figure S6). A similar estimate of surface silver atoms density is obtained from a topographical analysis in AFM (Figure 3). Inevitably both techniques makes assumptions, and the absolute accuracy of both estimates is therefore questionable. Nonetheless, there is a large discrepancy between the net charging of the 


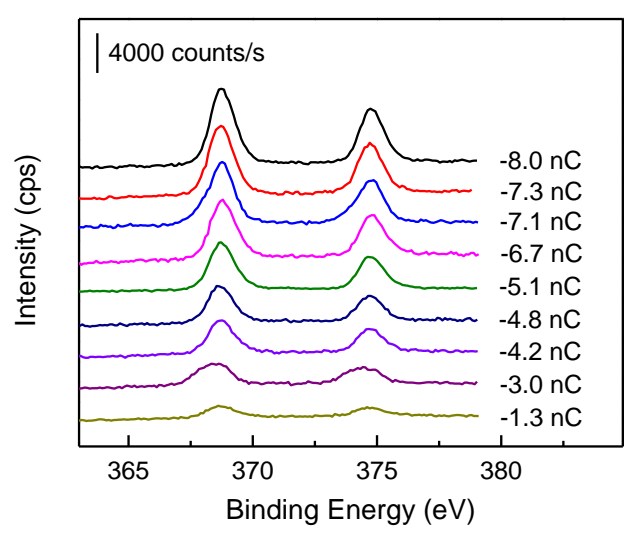

Figure 4. Electrochemical work by dielectrics scales with their net charging magnitude. Evolution of the XPS narrow scans signals for the silver $\mathrm{Ag} 3 \mathrm{~d}$ region of tribocharged PDMS samples that were discharged by immersion in a $5.0 \times 10^{-2} \mathrm{M}$ aqueous solution of $\mathrm{AgNO}_{3}$. The PDMS surface is charged by repeated contact against a glass surface and its net charging is measured prior to the metal discharge step using a Faraday pail connected to an electrometer. The sample initial charge-to-area ratio is indicated as label to the XPS trace. Curves are vertically offset for clarity; the absolute ordinate has no physical meaning.

sample and the extent to which metallic deposits grow - a Faraday pail measurement is a poor indicator on the electrochemical work that can be harvested from tribocharges. When XPS narrow scans of the Ag $3 \mathrm{~d}$ region for samples of different initial charge excess are compared, what becomes apparent for the first time, is that the electrochemical work done by charged dielectrics scales with their net charging magnitude (PDMS in Figure 4). This relationship further reinforces on a redox reaction being mediated by tribocharges. Further, the extent to which electrochemical work is in disproportion to the small net charging of the plastic disks parallels previous observation by KPFM of a mosaiclike $2 \mathrm{D}$ distribution of oppositely charged domains on the electrified surface of dielectrics. ${ }^{\mathrm{b}}$

If charged polymer fragments - previously tentatively described as "cryptoelectrons" work effector, the immediate implication in redox chemistry would be that the amount of work done by an electrified dielectric could largely exceed the Coulomb reading in the electrometer/Faraday pail. Our quantitative data on the reduction of silver ions align with this scenario. To further confirm this last point, we tested for silver deposition over Nylon samples bearing a net-positive charge. XPS data in Figure 5 show evidence of silver metal depositing on the polyamide surface even when the initial Faraday pail measurement indicates a net excess of positive charges. Our quantum computed value of work function for nylon is around $4.4 \mathrm{eV}$, which is analogous to the experimental value of 4.2-4.4 eV. ${ }^{34}$ The relatively small work function of nylon accounts for the positive reading by the electrometer.
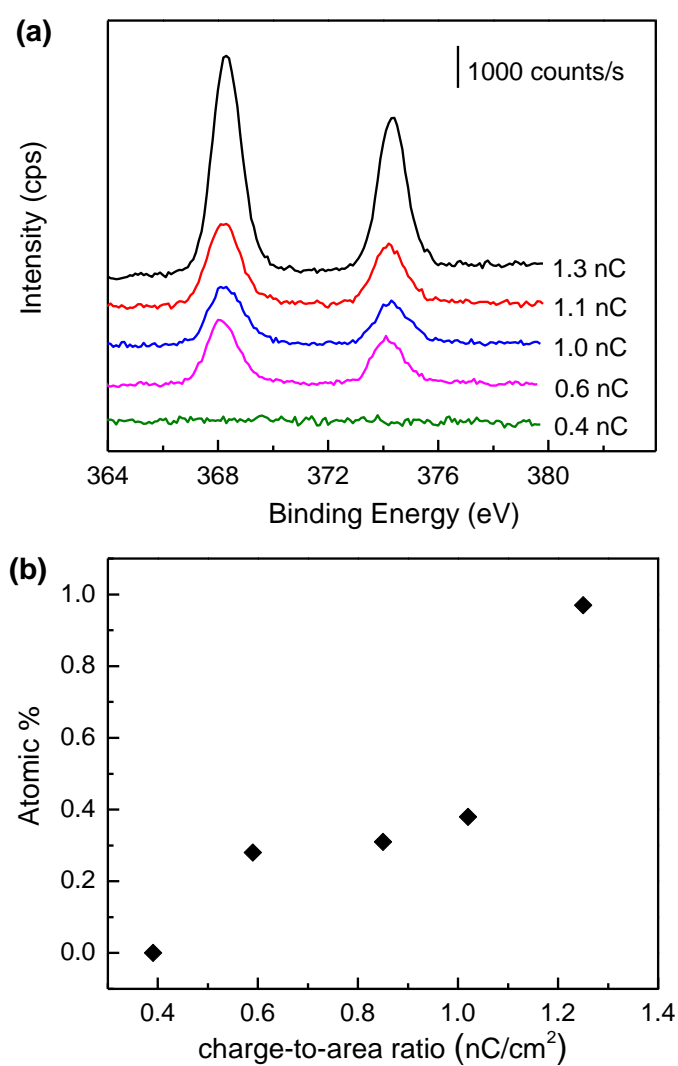

Figure 5. Silver ions reduction on positively charged nylon. (a) Narrow scans of the $\mathrm{Ag} 3 \mathrm{~d}$ region of tribocharged nylon samples that were discharged by immersing them in a 5.0 $\times 10^{-2} \mathrm{M}$ aqueous solution of $\mathrm{AgNO}_{3}$. Curves are vertically offset for clarity. (b) XPS-derived silver atomic percentages versus initial charge densities measured on nylon samples after the charge/discharge sequence.

The presence of metallic deposits after a discharge experiment in silver nitrate further reinforces on the simultaneous presence of both positive and negative surface domains. TEM can provide ex situ details on morphology and crystallinity of both the gold and silver particles while topographical AFM data (Figure 3) is obtained directly on the plastic sample after the discharge step. AFM data seem to indicate that while the growth of both silver and gold nanoparticles is overall homogeneous across the sample, there is evidence (Figure $3 \mathrm{a}, \mathrm{b}$ ) of nucleation being confined to sites of larger curvature. Diffusion of charges across the molecular chain of polyamides has been shown experimentally, ${ }^{25}$ most likely by involving a layer of water, 35 charges (electron as well as ions) can diffuse laterally on the surface of insulators, and electrons can travel up to $10 \mu \mathrm{m}$ across electron-beam irradiated insulators. ${ }^{36}$ Hence, in light of our AFM data and of these past reports, it is possible that charges flow from localized surface states to the particle's nucleation site, with the discharge process probably being initiated in regions of large charge density such at the top 
(a)

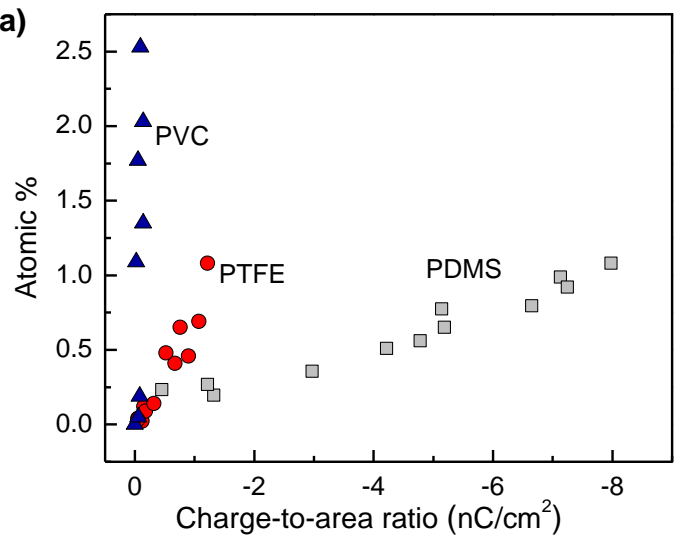

(b)

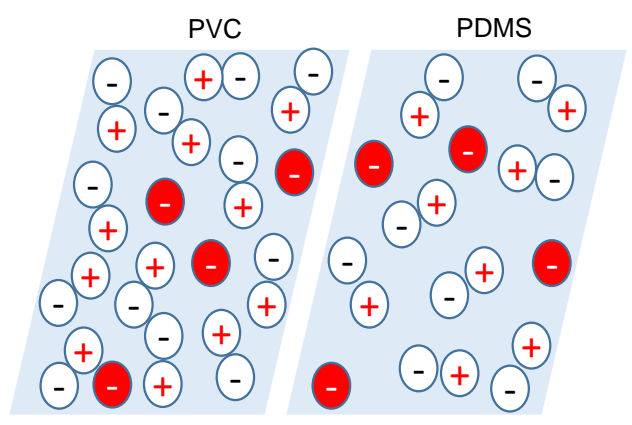

(c)

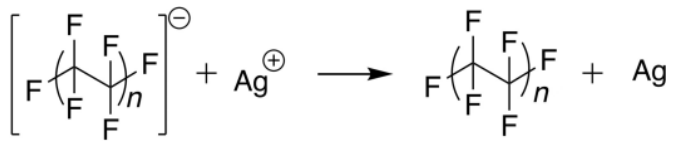

Figure 6. Material specific relationship between redox work and static charging (density) of dielectrics. (a) XPSdetermined amount of metallic silver that is deposited on plastic samples that are charged to a specific value of charge-to-area ratio by contact electrification against a glass surface. (b) Schematic depiction of a "mosaic" ensemble of tribocharges on two samples of equal charge excess. Due to a significant difference in ionization energy, PVC and PDMS samples of equal net charge density (number of red symbols) may have distinctly different total amount of negatively charged domains. (c) Outer sphere electron transfer reaction for the reduction of $\mathrm{Ag}^{+}$ions mediated by anion fragments on tribocharged PTFE.

of surface asperities. ${ }^{37}$ However, tests done on PVC, PTFE and PDMS samples having different roughness, did not show a link between the nanometer-size roughness probed by the AFM tip and the extent to which metal deposits (Supporting Information, Figure S7). The formation of silver nanoparticles via direct reduction of silver ions by anions derived from all of the polymers explored in this work is thermodynamically feasible: all the computed anions/neutral polymer redox potentials are all sufficiently negative to overcome the highly negative redox potential for isolated silver $\left(\mathrm{Ag}^{+} / \mathrm{Ag} ; E^{\mathrm{o}}=-1.8 \mathrm{~V}\right.$ vs $\left.\mathrm{SHE}^{28}\right) .^{38} \mathrm{Calcu}-$ lated reduction potentials in water of the species used in this study are $-4.05,-3.91,-4.44$, and $-3.89 \mathrm{~V}$ vs SHE for PVC, PDMS, PTFE and nylon, respectively (Table S1). 39 It indicates that the ability of a tribocharged insulator to trigger heterogeneous redox catalysis is more likely to be governed by a combination of work function and EA of the material rather than surface roughness and/or reducing power alone..$^{\circ 0}$ As can be inferred from the data in Figure 6, tribocharged dielectric surfaces yield the largest extent of electrochemical work when the IE of the dielectric is relatively small and the anions derived from the polymer are relatively unstable. PVC satisfies both these conditions and, as shown in Figure 6a, for any given value of net excess of negative charges being carried by the plastic ( $\mathrm{x}$-axis) PDMS and PTFE are less effective materials than PVC in the context of guiding a reductive heterogeneous redox process.

For these three different substrates the coverage of silver nanoparticles increases almost linearly with the charge density generated by contact electrification, but the three curves in Figure 6a do not run parallel to each other and are not separated by a vertical offset, as would be the case if only either EA or IE of the material were a key factor guiding metal ions discharge. Instead they depart with a material-specific slope from a common point located at the low end of the charge-to-area ratio scale.

Direct evidence of a material-specific relationship between static charging and attainable electrochemical work has never been reported and we propose that the root-cause behind the different slopes, at least for the non-branched polymers (PVC and PTFE), is a trade-off between the IE and EA of the polymer. PVC has the largest negative value of EA (Figure $1 \mathrm{~b}$ ) making negative charges unstable (Supporting Information, Figure $\mathrm{S}_{1}$ ) but at the same time it will ionize into cationic fragments with relative ease (Figure 1 a). This accounts for the generally limited extent to which PVC gains net-negative charging by means of contact electrification (blue symbols curve in Figure 6a) and suggests that for any negative value of charge-to-area ratio a large population of anions is required to offset cationic species. These anions are the effector of the redox work, hence this explains the largest values of silver atomic \% (y-axis) in Figure 6a. PTFE, on the other hand, showed a lower sensitivity in the electrochemical work versus static charging curve (red symbols in Figure 6a). This can also be accounted for in terms of the balance between IE and EA of the polymer. PTFE forms more stable anions and a small number of contact-separation cycles against glass leads immediately to a net excess of negative charges significantly larger to that achievable in PVC. Anions derived from PTFE are relatively stable and their absolute abundance at a given charge-toarea ratio is lower than in PVC since the ionization of PTFE to positively-charged species is not as favourable as for the former. The situation in PDMS and the small slope of the curve defined by the grey symbols in Figure 6 appears to be more complicated. The EA of PDMS is comparable to that of PTFE, with anionic species in both materials expected 
therefore to be of similar stability. Based on the computed values of IE, positive fragments should be even more abundant than for PVC, but surprisingly the PTFE curve in Figure 6 rests between the PVC and PDMS one.

\section{COUCLUSION}

This work shows that the discharge of metal ions over charged plastics result in nucleation of particles and points to anionic polymer fragments as being the mediators of the redox chemical changes. We bring circumstantial evidences for lateral movements of charges on dielectric surfaces towards the metal nanoparticle nucleation site and of inhomogeneous charge densities across the dielectric surface. PTFE, PDMS, PVC and nylon surface generate static charges by contact electrification, and regardless of the sign of the net charging all these dielectrics are capable of acting as electron sources and guide redox reactivity. There is however an imbalance between the net charging measured in a Faraday pail/electrometer set up and the extent to which the sample can act as electron source: it brings direct evidence for triboeletricity being a mosaic, ${ }^{\mathrm{b}}$ of positive and negative charges, rather than an homogenous ensemble. We bring evidence of a material-specific slope in the redox work vs static charge density curves, highlighting that a sensitivity factor has to be taken into account in the attempt of using plastics to deliver a specific amount of coulombs to a reaction mixture. Materials with a large negative EA and a relatively low IE - stable cations and unstable anions - can mediate redox work to a relatively large extent. Dielectric materials with stable anions - less negative EA - and large IE will probably be of greater importance when one attempts to deliver small redox changes with high precision. Taken together these findings constitute the most compelling evidence to date for tribocharges in polymers being ionic species. This work extends our understanıding of static electricity and may find applications in single-electrode electrochemistry and in the study of electrostatic catalysis on chemical reactivity. ${ }^{17,41}$

\section{EXPERIMENTAL SECTION}

Materials. Redistilled solvents and Milli- $\mathrm{Q}^{\mathrm{TM}}$ water $(>18$ $\mathrm{M} \Omega \mathrm{cm}$ ) were used for substrate cleaning and to prepare electrolytic solutions. Samples of PDMS (molecular weight of $500 \mathrm{kDa}, 840-5541$, RS Components Australia ${ }^{\circledR}$ ) and PVC (321-2105, RS Components Australia ${ }^{\circledR}$ ) were $1 \times 1 \mathrm{~cm}$ in size and approximately $0.3 \mathrm{~cm}$ in thickness. Samples of nylon (nylon-66, 514-607, RS Components Australia ${ }^{\circledR}$ ) and PTFE sheets were either of $1 \mathrm{~cm}$ radius and approximately $0.3 \mathrm{~cm}$ thick (68o-640, RS Components Australia ${ }^{\circledR}$ ) or disks 0.5 $\mathrm{mm}$ thick and $1.5 \mathrm{~cm}$ in radius and (Qorpak ${ }^{\circledR}$ ).

Charge Measurements. Plastic samples used for charge measurements and metal deposition (discharge) experiments were cleaned with water, methanol and dichloromethane, dried under a nitrogen flow, and then statically charged by contact. PDMS, PTFE and PVC samples were charged against a clean glass surface. The glass surface was cleaned by means of extensive washing with water, methanol and dichloromethane. Nylon disks were charged against a PDMS sample. Charged samples were handled in the dark and the magnitude of their net charge was varied either by changing the number of contact-separation cycles or by changes to the time elapsed between contact and separation. The charge of the sample was measured by an electrometer and Faraday pail set-up (JCI 140 static monitor and JCI 147 charge measurement units), purchased and calibrated by DEKRA (Southampton Science Park, Southampton, UK), and operating on the $10^{-9}$ Coulomb scale. Charging data are reported as charge-to-geometric area ratios. The precision of the technique, as estimated by standard deviation, is $95 \%$ (or less) of multiple readings on the same sample.

Metal ions discharge experiments and surface characterization. Tribocharged plastic samples were transferred to a $50 \mathrm{mM}$ aqueous solution of $\mathrm{AgNO}_{3}$ (99.999\%, Sigma), $\mathrm{HAuCl}_{4}$ (99.99\%, Sigma), $\mathrm{CuSO}_{4}$ (99.99\%, Sigma) and $\mathrm{PbCl}_{2}$ (99.99\%, Sigma) rested for $3 \mathrm{~h}$ in the liquid in a dark environment, washed with Milli- $\mathrm{Q}^{\mathrm{TM}}$ water, and then dried under a stream of nitrogen before X-ray photoelectron spectroscopy. X-ray photoelectron spectroscopy (XPS) characterization was performed on an ESCALab $250 \mathrm{Xi}$ (Thermo Scientific) spectrometer with a monochromated $\mathrm{Al} \mathrm{K} \alpha$ source. The pressure in the analysis chamber during measurement was $<10^{-8}$ mbar. The pass energy and step size for narrow scans were $20 \mathrm{eV}$ and $0.1 \mathrm{eV}$ respectively, with a take-off angle normal to the sample surface. Spectral analysis was performed using Avantage 4.73 software and background spectra were considered as Smart type, and curve fitting used functions with a Gaussian-Lorentzian character. Elemental sensitivity factors for the $\mathrm{C}$ is, $\mathrm{N}$ is, $\mathrm{O}$ 1s, $\mathrm{F} 1 \mathrm{~s}, \mathrm{Cl}$ 2p, $\mathrm{Si} 2 \mathrm{p}, \mathrm{Au} 4 \mathrm{f}$ and $\mathrm{Ag} 3 \mathrm{~d}$ regions are 1.00o, 1.676, 2.881, 4.118, 2.741, 0.900, 20.735 and 22.131, respectively. Binding energies obtained from high-resolution scans were calibrated by applying a rigid shift to bring the main $C$ is emission to $284.8 \mathrm{eV}$. XPS experiments for the data in Figure S9 were performed on an ESCALab 250 Xi (Thermo Scientific) spectrometer with a monochromated $\mathrm{Al} \mathrm{K \alpha}$ source. The time that elapsed between the Faraday pail measurement and the immersion of the tribocharged sample in the liquid was $60 \mathrm{~s}$ or less. The charge loss in ambient air over this period is negligible (Supporting Information, Figure $\mathrm{S} 1$ ). Low-resolution transmission electron microscopy (TEM) was conducted using a JEOL 2100 microscope with an accelerating voltage of $120 \mathrm{kV}$. High-resolution TEM (HRTEM) and selected area electron diffraction (SAED) were performed on a FEI Titan G2 8o-20o microscope operating at $200 \mathrm{kV}$ at room temperature. The TEM support grid was copper and metal deposited on the mesh of the grid was prepared as follows: firstly, a large PDMS sample $(50 \times 10 \mathrm{~cm})$ was charged by contacting it against a clean glass, then immediately immersed in an aqueous solution (ca. $50 \mathrm{~mL}$ ) of either $\mathrm{AgNO}_{3}(50 \mathrm{mM})$ or $\mathrm{HAuCl}_{4}(50 \mathrm{mM})$ for $3 \mathrm{~h}$ and completely shielded from ambient light. The PDMS sheet was then removed from the solution, washed 
extensively with water, dried under a stream of nitrogen, rolled up and immersed in water (ca. $100 \mathrm{~mL}$ ) and sonicated for $15 \mathrm{~min}$. The water sample was then passed several times over the grid before TEM and HRTEM analysis. Measurements of sample roughness (Supporting Information, Figure $\mathrm{S}_{7}$ ) were obtained from Atomic force microscopy (AFM) experiments performed in tapping mode, using a Dimension FastScan (Bruker Corporation, Santa Barbara, CA, USA). They are reported as root mean square surface roughness $\left(R_{\mathrm{q}}\right)$, average surface roughness $\left(R_{\mathrm{a}}\right)$, and as values of maximum roughness $\left(R_{\max }\right)$. AFM imaging was done in air, at room temperature, and by using TESPA-V2 probes with a spring constant of $42 \mathrm{~N} \mathrm{~m}^{-1}$. The imaging resolution was set to 256 points/line, the scan rate to $1.00 \mathrm{~Hz}$ and the peak force to $20 \mathrm{nN}$. KPFM (Kelvin probe force measurements) were performed on a Bruker Dimension Icon Scanning Probe Microscope in KPFM-AM mode. Pt tips (RMN-25 $\mathrm{PT} 300 \mathrm{~B})$ were purchased from RMN Probes (tip radius curvature $<20 \mathrm{~nm}$, spring constant $18 \mathrm{~N} \mathrm{~m}^{-1}$, resonant frequency of $20 \mathrm{kHz}$ ).

Theoretical procedures. Ionization energies and electron affinities of oligomeric polymer chains of varying lengths were carried out using standard density functional theory at the Mo62X/6-31+G(d) level of theory. This level of theory has previously been shown to provide sufficiently accurate predictions of such quantities. ${ }^{42}$ Because the electrons are gained or lost at the solid-air interface, the polymer systems were treated as being in the gas phase, and thermal corrections to $298.15 \mathrm{~K}$ were evaluated using the standard textbook formulae for an ideal gas under the harmonic oscillator-rigid rotor approximation. All calculations were carried out using Gaussian 16 software $^{43}$ and all structures considered are shown in Supporting Information, Figure S3. In the case of PDMS, the polymer is a cross-linked network and so linear, mono-substituted (mono-PDMS) and di-substitued chains (di-PDMS) were studied. The chain length was defined so that a consistent number of repeated units (n) is compared. For the $n=1$ species, the reduction potentials in water versus SHE were also computed. For this purpose, solvation energies of the $n=1$ polymer and polymer anions in water were calculated with SMD at the B3-LYP/6-31+G(d) level of theory, ${ }^{44}$ while the electron was treated under the electron convention with Fermi-Dirac statistics $(-3.62 \mathrm{~kJ} / \mathrm{mol})$. The resulting Gibbs free energy of reduction was then converted to a reduction potential versus SHE using the Nernst equation and a value of the SHE reduction potential of 3.91V.45 Further details and all raw data are provided in the supporting information.

\section{ASSOCIATED CONTENT}

Supporting Information

The Supporting Information is available free of charge on the ACS Publications website.

Detailed computational methodology, additional theoretical calculations, XPS, AFM, KPFM, TEM and SAED spectra data.

\section{AUTHOR INFORMATION}

\section{Corresponding Authors}

michelle.coote@anu.edu.au

simone.ciampi@curtin.edu.au

\section{ACKNOWLEDGMENTS}

This work was financially supported by grants from the Australian Research Council (DP190100735 (S.C and N.D.), DE160100732 (S.C.), DE160101101 (N.D.), DE160100589 (G.J.), FL170100041 and CE140100012 (M.L.C)). The authors acknowledge the use of Curtin University's Microscopy \& Microanalysis Facility, whose instrumentation has been partially funded by the University, State and Commonwealth. M.L.C. acknowledges allocations of supercomputing time on the $\mathrm{Na}-$ tional Facility of the Australian National Computational Infrastructure.

\section{REFERENCES}

(1) Loveland, R. J. Electrostatic Ignition Hazards in Industry. J. Electrost. 1981, 11, 3-11.

(2) Childress, C. O.; Kabell, L. J., Electrostatic printing system. Google Patents: 1963.

(3) (a) Lowell, J.; Rose-Innes, A. C. Contact Electrification. Adv. Phys. 1980, 29, 947-1023. (b) Diaz, A. F.; Guay, J. Contact Charging of Organic Materials: Ion vs. Electron Transfer. IBM J. Res. Dev. 1993, 37, 249-260. (c) Davies, D. K. Charge Generation on Dielectric Surfaces. J. Phys. D: Appl. Phys. 1969, 2, 1533-1537.

(4) Gibson, H. W.; Pochan, J. M.; Bailey, F. Surface Analyses by a Triboelectric Charging Technique. Anal. Chem. 1979, 51, 483-487.

(5) de Lima Burgo, T. A.; Rezende, C. A.; Bertazzo, S.; Galembeck, A.; Galembeck, F. Electric Potential Decay on Polyethylene: Role of Atmospheric Water on Electric Charge Build-Up and Dissipation. $J$. Electrost. 2011, 69, 401-409.

(6) Camara, C. G.; Escobar, J. V.; Hird, J. R.; Putterman, S. J. Correlation Between Nanosecond X-ray Flashes and Stick-slip Friction in Peeling Tape. Nature 2008, 455, 1089-1092.

(7) Cress, G. O.; Brady, B. T.; Rowell, G. A. Sources of Electromagnetic Radiation from Fracture of Rock Samples in the Laboratory. Geophys. Res. Lett. 1987, 14, 331-334.

(8) (a) Galembeck, F.; Burgo, T. A. L.; Balestrin, L. B. S.; Gouveia, R. F.; Silva, C. A.; Galembeck, A. Friction, Tribochemistry and Ttriboelectricity: Recent Progress and Perspectives. RSC Adv. 2014, 4, 64280-64298. (b) Williams, M. W. Triboelectric Charging of Insulating Polymers-Some New Perspectives. AIP Adv. 2012, 2, 010701.

(9) (a) Burgo, T. A. L.; Ducati, T. R. D.; Francisco, K. R.; Clinckspoor, K. J.; Galembeck, F.; Galembeck, S. E. Triboelectricity: Macroscopic Charge Patterns Formed by Self-Arraying Ions on Polymer Surfaces. Langmuir 2012, 28, 7407-7416. (b) Baytekin, H. T.; Patashinski, A. Z.; Branicki, M.; Baytekin, B.; Soh, S.; Grzybowski, B. A. The Mosaic of Surface Charge in Contact Electrification. Science 2011, 333, 308-312.

(10) Liu, C.-Y.; Bard, A. J. Chemical Redox Reactions Induced by Cryptoelectrons on a PMMA Surface. J. Am. Chem. Soc. 2009, 131, 63976401.

(11) Lazić, P.; Persson, B. N. J. Surface-Roughness-Induced Electric-Field Enhancement and Triboluminescence. EPL 2010, 91, 46003-46007.

(12) Wu, C.; Kim, T. W.; Park, J. H.; An, H.; Shao, J.; Chen, X.; Wang, Z. L. Enhanced Triboelectric Nanogenerators Based on $\mathrm{MoS}_{2}$ Monolayer Nanocomposites Acting as Electron-Acceptor Layers. ACS Nano 2017, 11, 8356-8363.

(13) Rosenblum, B.; Bräunlich, P.; Himmel, L. Spontaneous Emission of Charged Particles and Photons During Tensile Deformation of OxideCovered Metals under Ultrahigh-Vacuum Conditions. J. Appl. Phys. 1977, $48,5262-5273$.

(14) Kim, S.; Ha, J.; Kim, J.-B. The Effect of Dielectric Constant and Work Function on Triboelectric Nanogenerators: Analytical and Numerical Study. Integr. Ferroelectr. 2016, 176, 251-256. 
(15) Li, A.; Zi, Y.; Guo, H.; Wang, Z. L.; Fernández, F. M. Triboelectric Nanogenerators for Sensitive Nano-Coulomb Molecular Mass Spectrometry. Nat. Nanotechnol. 2017, 12, 481-487.

(16) (a) Baytekin, B.; Baytekin, H. T.; Grzybowski, B. A. What Really Drives Chemical Reactions on Contact Charged Surfaces? J. Am. Chem. Soc. 2012, 134, 7223-7226. (b) Liu, C.-Y.; Bard, A. J. Electrostatic Electrochemistry at Insulators. Nat. Mater. 2008, 7, 505-509. (c) Liu, C.Y.; Bard, A. J. Electrostatic Electrochemistry: Nylon and Polyethylene Systems. Chem. Phys. Lett. 2010, 485, 231-234.

(17) (a) Ciampi, S.; Darwish, N.; Aitken, H. M.; Díez-Pérez, I.; Coote, M. L. Harnessing Electrostatic Catalysis in Single Molecule, Electrochemical and Chemical Systems: a Rapidly Growing Experimental Tool Box. Chem. Soc. Rev. 2018, 47, 5146-5164. (b) Shaik, S.; Ramanan, R.; Danovich, D.; Mandal, D. Structure and Reactivity/Selectivity Control by OrientedExternal Electric Fields. Chem. Soc. Rev. 2018, 47, 5125-5145.

(18) Terris, B. D.; Stern, J. E.; Rugar, D.; Mamin, H. J. Contact Electrification Using Force Microscopy. Phys. Rev. Lett. 1989, 63, 26692672

(19) Nakajima, A.; Miyahara, H.; Ishikawa, T.; Wada, J.; Yanabu, S. Streaming Electrification Characteristics of Silicone Oil. IEEE Trans. Dielectr. Electr. Insul. 2008, 15, 519-526.

(20) Park, J. Y.; Salmeron, M. Fundamental Aspects of Energy Dissipation in Friction. Chem. Rev. 2013, 114, 677-711.

(21) Dickinson, J. T.; Donaldson, E. E.; Park, M. K. The Emission of Electrons and Positive Ions From Fracture of Materials. J. Mater. Sci. 1981, $16,2897-2908$.

(22) Fischer, T. E. Tribochemistry. Ann. Rev. Mater. Sci. 1988, 18, 303 323.

(23) (a) Li, Y.; Haworth, N. L.; Xiang, L.; Ciampi, S.; Coote, M. L.; Tao, N. Mechanical Stretching-Induced Electron-Transfer Reactions and Conductance Switching in Single Molecules. J. Am. Chem. Soc. 2017, 139, 14699-14706. (b) Ladenthin, J. N.; Frederiksen, T.; Persson, M.; Sharp, J. C.; Gawinkowski, S.; Waluk, J.; Kumagai, T. Force-Induced Tautomerization in a Single Molecule. Nat. Chem. 2016, 8, 935-940.

(24) Gallo, C.; Lama, W. Classical Electrostatic Description of the Work Function and Ionization Energy of Insulators. IEEE Trans. Ind. Appl. 1976, 12, 7-11.

(25) Pandey, P. C.; Shukla, S.; Pandey, Y. 3-Aminopropyltrimethoxysilane and Graphene Oxide/Reduced Graphene Oxide-Induced Generation of Gold Nanoparticles and Their Nanocomposites: Electrocatalytic and Kinetic Activity. RSC Adv. 2016, 6, 80549-80556.

(26) Kathmann, S. M.; Kuo, I.-F. W.; Mundy, C. J. Electronic Effects on the Surface Potential at the Vapor-Liquid Interface of Water. J. Am. Chem. Soc. 2008, 130, 16556-16561.

(27) Lee, J. K.; Samanta, D.; Nam, H. G.; Zare, R. N. Spontaneous Formation of Gold Nanostructures in Aqueous Microdroplets. Nat. Соттип. 2018, 9, 1562-1570.

(28) Gentry, S. T.; Fredericks, S. J.; Krchnavek, R. Controlled Particle Growth of Silver Sols Through the Use of Hydroquinone as a Selective Reducing Agent. Langmuir 2009, 25, 2613-2621.

(29) Henglein, A. Non-Metallic Silver Clusters in Aqueous Solution: Stabilization and Chemical Reactions. Chem. Phys. Lett. 1989, 154, 473476.

(30) (a) Jafarkhani, P.; Torkamany, M. J.; Dadras, S.; Chehrghani, A.; Sabbaghzadeh, J. Necklace-Shaped Au-Ag Nanoalloys: Laser-Assisted Synthesis and Nonlinear Optical Properties. Nanotechnology 2011, 22, 235703-235709. (b) Romand, M.; Roubin, M.; Deloume, J.-P. X-ray Photoelectron Emission Studies of Mixed Elenides $\mathrm{AgGaSe}_{2}$ and $\mathrm{Ag}_{9} \mathrm{GaSe}_{6}$ J. Solid State Chem. 1978, 25, 59-64.

(31) (a) Reshetenko, T. V.; St-Pierre, J.; Bethune, K. P.; Artyushkova, K.; Rocheleau, R.; Atanassov, P. Multi-Analytical Study of Gas Diffusion Layer PTFE Content Local Variation. ECS Trans. 2013, 50, 591-599. (b) Hunke, H.; Soin, N.; Shah, T. H.; Kramer, E.; Pascual, A.; Karuna, M. S. L.; Siores, E. Low-Pressure $\mathrm{H}_{2}, \mathrm{NH}_{3}$ Microwave Plasma Treatment of Polytetrafluoroethylene (PTFE) Powders: Chemical, Thermal and Wettability Analysis. Materials 2015, 8, 2258-2275.

(32) (a) Wu, Q.; Diao, P.; Sun, J.; Jin, T.; Xu, D.; Xiang, M. Electrodeposition of Vertically Aligned Silver Nanoplate Arrays on Indium Tin Oxide Substrates. J. Phys. Chem. C 2015, 119, 20709-20720. (b) Yao, Q.; Fan, B.; Xiong, Y.; Wang, C.; Wang, H.; Jin, C.; Sun, Q. Stress Sensitive Electricity Based on Ag/Cellulose Nanofiber Aerogel for SelfReporting. Carbohydr. Polym. 2017, 168, 265-273.
(33) Pootawang, P.; Saito, N.; Takai, O.; Lee, S.-Y. Synthesis and Characteristics of $\mathrm{Ag} / \mathrm{Pt}$ Bimetallic Nanocomposites by Arc-Discharge Solution Plasma Processing. Nanotechnology 2012, 23, 395602-395609.

(34) Arridge, R. G. C. The Static Electrification of Nylon 66. Br. J. Appl. Phys. 1967, 18, 1311-1316.

(35) Awakuni, Y.; Calderwood, J. H. Water Vapour Adsorption and Surface Conductivity in Solids. J. Phys. D: Appl. Phys. 1972, 5, 1038-1045. (36) Sessler, G. M.; Figueiredo, M. T.; Ferreira, G. F. L. Models of Charge Transport in Electron-Beam Irradiated Insulators. IEEE Trans. Dielectr. Electr. Insul. 2004, 11, 192-202.

(37) Shaik, S.; Mandal, D.; Ramanan, R. Oriented Electric Fields as Future Smart Reagents in Chemistry. Nat. Chem. 2016, 8, 1091-1098.

(38) XPS data in Figure S8 show that tribocharged PVC samples were able to mediate the reduction of $\mathrm{Cu}^{2+}$ ions to metallic copper, but unable to reduce $\mathrm{Pb}^{2+}$ (from $5.0 \times 10^{-2} \mathrm{M}$ aqueous solutions of $\mathrm{CuSO}_{4}$ and $\mathrm{PbCl}_{2}$, respectively).

(39) Reduction potentials for the polymers converge to approximately $-3.66,-3.74,-2.68$, and $-2.84 \mathrm{~V}$ vs SHE (Table S1).

(40) Analysis of morphology and metal deposition (XPS) on PTFE sample of similar charging magnitude but sourced from different suppliers is in Figure $\mathrm{S} 9$.

(41) Liu, C.-Y.; Bard, A. J. Electrochemistry and Electrogenerated Chemiluminescence with a Single Faradaic Electrode. Anal. Chem. 2005, $77,5339-5343$.

(42) Zhao, Y.; Truhlar, D. G. The M06 Suite of Density Functionals for Main Group Thermochemistry, Thermochemical Kinetics, Noncovalent Interactions, Excited States, and Transition Elements: Two New Functionals and Systematic Testing of Four M06-class Functionals and 12 Other Functionals. Theor. Chem. Acc. 2008, 120, 215-241.

(43) Frisch, M.; Trucks, G.; Schlegel, H.; Scuseria, G.; Robb, M.; Cheeseman, J.; Scalmani, G.; Barone, V.; Petersson, G.; Nakatsuji, H. Gaussian 16, revision A. 03. Gaussian Inc., Wallingford CT 2016.

(44) Marenich, A. V.; Ho, J.; Coote, M. L.; Cramer, C. J.; Truhlar, D. G. Computational Electrochemistry: Prediction of Liquid-Phase Reduction Potentials. Phys. Chem. Chem. Phys. 2014, 16, 15068-15106.

(45) Isse, A. A.; Gennaro, A. Absolute Potential of the Standard Hydrogen Electrode and the Problem of Interconversion of Potentials in Different Solvents. J. Phys. Chem. B 2010, 114, 7894-7899. 


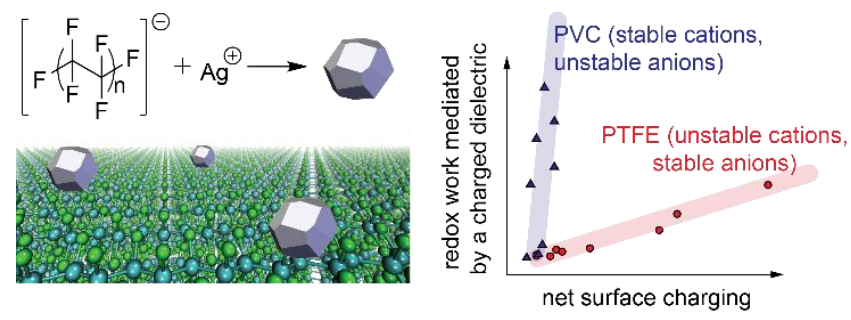

